\title{
A CASE OF EUGLYCAEMIC DIABETIC KETOACIDOSIS IN A PATIENT TREATED WITH CANOGLIFLOZIN
}

\section{Abstract}

Background: Canagliflozin is an oral hypoglycaemic agent from the novel class of Sodium Glucose Co-Transporter 2 (SGLT-2) inhibitors, used in the treatment of patients with Type 2 Diabetes Mellitus (T2DM). Although effective in the treatment of hyperglycaemia, these medications have been linked to the development of diabetic ketoacidosis (DKA). We describe the case of a patient with T2DM who presented with severe metabolic acidosis while taking Canagliflozin

Case: We report the case of a 43-year-old Caucasian male with T2DM who presented with vomiting, dehydration, fatigue and abdominal pain. He had been prescribed Canagliflozin four months earlier. The patient was found to have a severe metabolic acidosis, with high urinary ketones but normal blood glucose levels. He was haemodynamically stable at presentation, and remained so throughout admission. Treatment with intravenous insulin, fluids and sodium bicarbonate resolved the acidosis, and canagliflozin was stopped.

Discussion: SGLT-2 inhibitors prevent glucose resorption from urine, leading to increased urinary glucose clearance and subsequent improvement of glycaemic control. Nonetheless, in May 2015, the FDA published a safety warning for this class of drugs, reporting over 20 cases of DKA in patients taking the medication. It is important for clinicians and patients to be aware of the potential risk of euglycaemic DKA in patients taking SGLT2 inhibitors. Acute illness, dehydration and relative insulinopaenia may be predisposing factors. Whether supplying patients prescribed these medications with ketone meters could help prevent DKAs, or lead to earlier admissions, merits further research.

\section{Introduction}

Canagliflozin, of the Sodium Glucose Co-Transporter 2 (SGLT-2) inhibitors class of oral hypoglycaemic agents, is used in the treatment of Type 2 Diabetes Mellitus (T2DM). SGLT-2 inhibitors work by lowering the renal threshold for glucose excretion, resulting in a greater urinary excretion of glucose. Canagliflozin was approved for use in both the EU and USA in 2013. In the UK, the National Institute for Health and Care Excellence (NICE) state that it can be used as the first stage of treatment intensification in T2DM ${ }^{1}$, following use of metformin.

In May 2015, the FDA published a safety warning ${ }^{2}$ for SGLT-2 inhibitors, reporting over twenty cases of diabetic ketoacidosis (DKA) in patients taking the medication. We report the case of a 43-year-old Caucasian male who presented to $A \& E$ four months following initiation of treatment with canagliflozin.

\section{The Case}

The patient was a 43-year-old Caucasian male with T2DM. His blood glucose levels were previously well controlled with metformin, sitagliptin, gliclazide and canagliflozin. His BMI was 21.8. He was haemodynamically stable at presentation, and throughout his admission.

\section{Symptoms:}

- Three days of fatigue, loss of appetite, and generalised aches and pains

- One episode of vomiting on the day of presentation

- Several episodes of diarrhoea four days earlier, with severe right-sided abdominal pain on the day of presentation. He also reported symptoms that were in keeping with ileus.

References

1. Type 2 diabetes in adults: management. NICE guidelines [NG28]. Published December 2015. Lasted updated July 2016 Accessible at $\langle$ https://www.nice.org.uk/guidance/ng28>

2. FDA Drug Safety Communication: FDA warns that SGLT2 inhibitors for diabetes may result in a serious condition of too much acid in the blood. May 5 2015. The FDA. Accessible at http://www.fda.gov/Drugs/DrugSafety/ucm446845.htm. Last accessed 27/10/16

\begin{tabular}{|l|c|c|}
\hline \multicolumn{2}{|c|}{ Investigations } \\
\hline Urine & Glucose & 1000 \\
\hline & Ketones & $150(+++)$ \\
\hline Arterial Blood Gas & Protein & 75 \\
\hline & $\mathrm{pH}$ & 7.051 \\
\hline & Glucose $(\mathrm{mmol} / \mathrm{L})$ & 9.5 \\
\hline Biochemistry & Base Excess & -25.2 \\
\hline & Anion Gap & 20.8 \\
\hline & $\mathrm{Na}(\mathrm{mmol} / \mathrm{L})$ & 131 \\
\hline & $\mathrm{K}(\mathrm{mmol} / \mathrm{L})$ & 3.3 \\
\hline & $\mathrm{U}(\mathrm{mmol} / \mathrm{L})$ & 3.4 \\
\hline
\end{tabular}

Treatment

- Transferred to ITU, and treated with intravenous fluids, insulin, and sodium bicarbonate. Canagliflozin was stopped. The acidosis resolved, and regular insulin commenced.

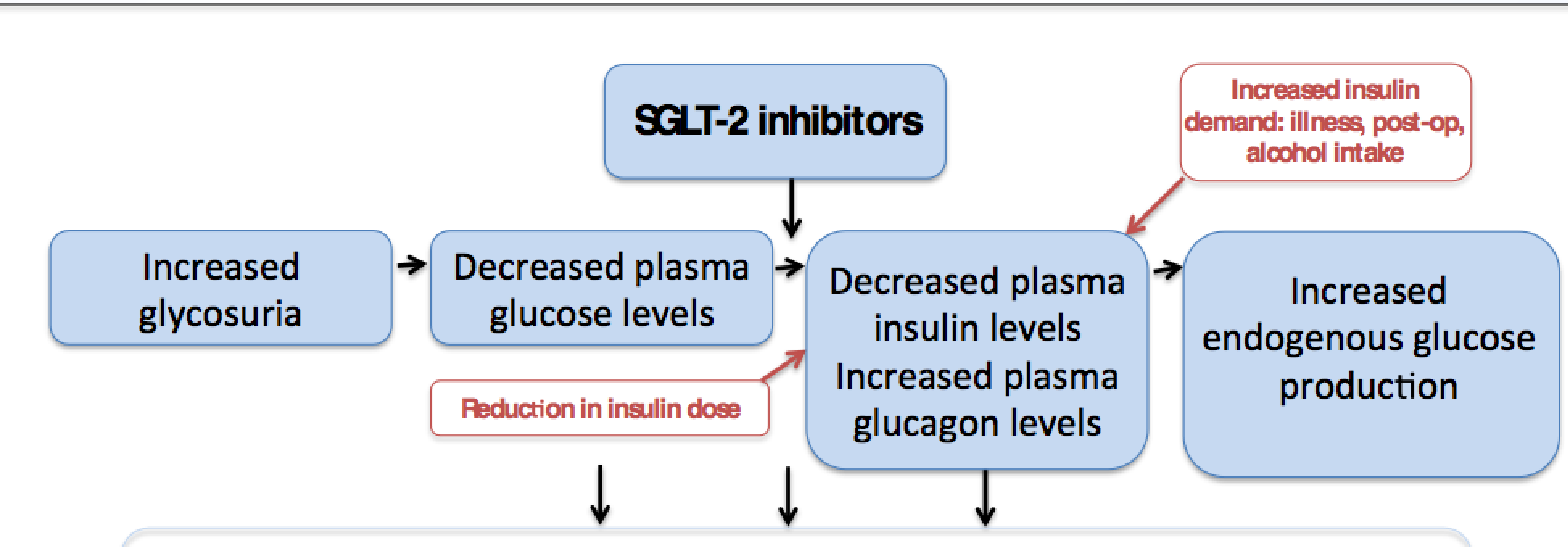

Milder insulin deficiency, milder insulin resistance $=$ lower rate of glucose overproduction and under-utilisation than in DKA but with increased urinary

glucose clearance

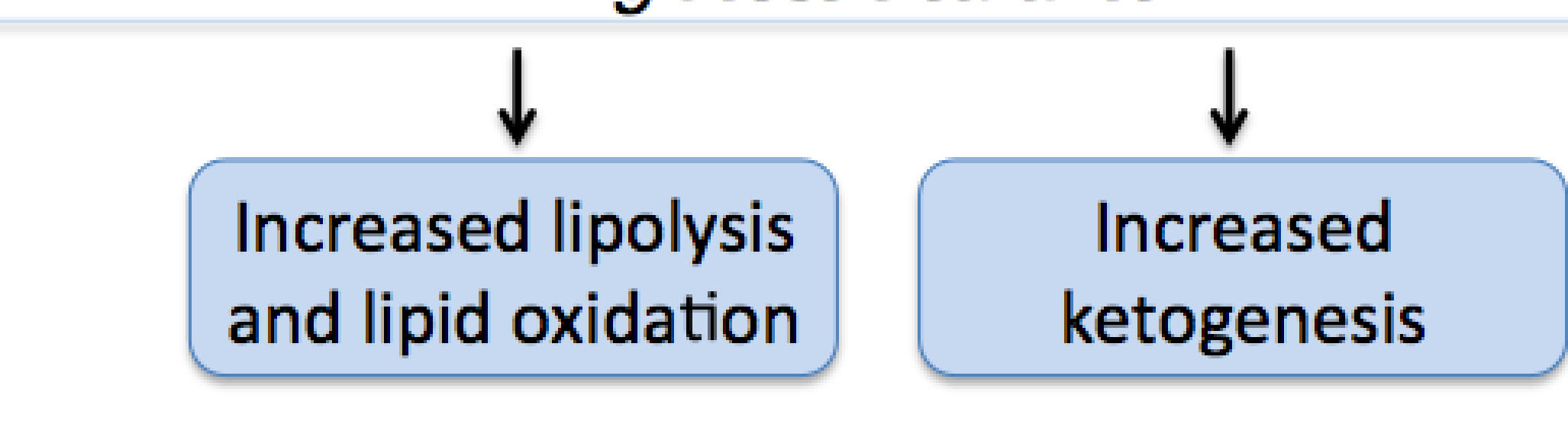

Malaise leads to checking of blood sugar, which is within normal limits $\downarrow$

euDKA

\section{Discussion}

To our knowledge this is the first reported case in the UK of a patient presenting with euglycaemic DKA caused by treatment with SGLT-2 inhibitors.

Cases of euglycaemic diabetic ketoacidosis (euDKA) have been reported in patients taking canagliflozin ${ }^{3,4}$. Increased urinary excretion of glucose lowers plasma glucose levels, and predisposes the patient to a ketogenic state. Since blood glucose levels are within range, and unless patients monitor ketone levels, they may not seek medical attention despite feeling profoundly unwell. Predisposing factors are thought to include acute illness, dehydration, periods of fasting, and relative insulinopaenia ${ }^{5}$.

It is important for both clinicians and patients to be aware of the risk of euDKA when taking SGLT-2 inhibitors, as acidosis may develop insidiously. Provision of ketone-meters to patients prescribed this class of medication may prevent development of euDKA, or lead to earlier admissions to hospital. This warrants further research. Patients should be educated about the risks of taking an SGLT-2 inhibitor, and the possibility that they may become unwell while still having an acceptable plasma glucose.

3. Peters AL, Buschur EO, Buse JB, Cohan P, Diner JC and Hirsch IB. Euglycaemic diabetic ketoacidosis: a potential complication of treatment with sodium-glucose cotransporter 2 ketoacidosis: a potential complication of treatment $w$

in

Condu N, Desai M, Ways K and Meininger G. Diabetic ketoacidosis and related events in the

canagliflozin Type 2 diabetes clinical program. Diabetes Care $2015 \mathrm{Jul}$.

5. Rosenstock J and Ferrannini E. Euglycaemic diabetic keoacidosis: a predictable, detectable, and preventable safety concern with SGLT2 inhibitors. Diabetes Care 2015 Sep; 38 (9): 1638-
1642. 